\title{
Favorable clinical efficacy of mepolizumab on the upper and lower airways in severe eosinophilic asthma: a 48-week pilot study
}

\author{
${ }^{1}$ Department of Allergy and Respiratory Medicine, Sutoh Hospital, Annaka, Gunma, Japan \\ ${ }^{2}$ Department of Radiology, Sutoh Hospital, Annaka, Gunma, Japan
}

\section{KEY WORDS}

eosinophilic asthma; forced expiratory volume in one second; Lund-MacKay CT scoring;

mepolizumab; SNOT-22 score

\section{Corresponding author}

Motohiro Kurosawa

Department of Allergy and Respiratory Medicine

Sutoh Hospital, 3532-5 Annaka,

Gunma 379-0116, Japan

Phone: +81273823131

Fax: +81273826568

E-mail: motohiro@kl.wind.ne.jp

Doi

10.23822/EurAnnACI.1764-1489.94

\begin{abstract}
Summary
Objective. Assessing efficacy of mepolizumab on the upper and lower airways in severe eosinophilic asthma patients. Patients and methods. This study was a 48-week prospective open-label analysis of mepolizumab in 11 asthmatics with chronic rhinosinusitis (CRS). It was administered every 4 weeks. Six patients were aspirin-exacerbated respiratory disease (AERD). Results. Blood eosinophil count was reduced after the first administration, and was continued until 48 weeks. The Sino-Nasal Outcome Test scores, the Lund-MacKay CT scoring, and forced expiratory volume in 1 second were improved. Symptom scores of anosmia and nasal congestion were not improved in the patients with AERD. All oral corticosteroid-dependent patients successfully withdrew from corticosteroids. Conclusions. This pilot study showed mepolizumab improved nasal symptoms and lung function in severe eosinophilic asthma patients with CRS, suggesting efficacy of mepolizumab on the upper and lower airway symptoms in eosinophilic asthma.
\end{abstract}

\section{Introduction}

It has been clearly demonstrated that the upper and lower airway diseases share common immunopathological mechanisms $(1,2)$. The term "one airway disease" has been established between not only allergic rhinitis, but also chronic rhinosinusitis (CRS) and asthma $(3,4)$. Clinical relationship between CRS and asthma has been a growing health concern, and reported incidence of asthma is at least $50 \%$ in patients with CRS (5).

Current consensus in Europe and the United States discerns 2 major phenotypes defined as subgroups of patients homogeneous clinically observations: CRS with nasal polyps and CRS without nasal polyps (6,7). The former in white patients is characterized by eosinophilic inflammation with high interleukin 5 (IL-5) level in the tissue (8). Also, investigations of cytokine profiles in Japanese patients with CRS demonstrated that eosinophilic infiltration was a common histological fea- ture, and cytokine profiles in the Japanese resembled those in Europe and the United States (9-11).

Because IL-5 plays a key role on chemotaxis, differentiation, activation, and survival of eosinophils (12), and because those cells represent such prominent characteristics in the polyps, antagonism of IL-5 has been considered a therapeutic target. The first pilot study with reslizumab, a humanized IL-5 antibody, showed a significant reduction of the size of nasal polyps after a single intravenous injection (13). Hence, the principle of IL-5 antagonism was established in eosinophilic nasal polyps.

Mepolizumab, a humanized $\mathrm{IgG}_{1}$ monoclonal antibody that blocks human IL-5 from binding to the IL-5 receptor, has been shown to be a potential novel therapeutic approach in patients with severe eosinophilic nasal polyposis (14). An international randomized, double-blinded, placebo-controlled, multicenter study including 105 patients treated with mepolizumab or placebo showed that in patients with recurrent nasal polyposis re- 
ceiving topical corticosteroids, mepolizumab treatment led to a greater reduction in the need of surgery and a greater improvement on symptoms than placebo (15).

Aspirin-exacerbated respiratory disease (AERD) is the triad of CRS with nasal polyposis, adverse reaction to aspirin, and asthma (16). A recent retrospective analysis of mepolizumab in 22 patients with AERD provided clinical evidences that IL-5 inhibition improved subject-reported upper and lower airway symptoms, but not improved forced expiratory volume in 1 second $\left(\mathrm{FEV}_{1}\right)(17)$. To our knowledge, no data has been reported on the benefit of mepolizumab in severe eosinophilic asthma patients with CRS. This is the first prospective open-label pilot study of 48-week subcutaneous administration of mepolizumab in Japanese patients of severe eosinophilic asthma with CRS. The primary objective of this study was to investigate whether mepolizumab treatment may improve the symptoms of CRS and the findings of computed tomography (CT) scan opacification of paranasal sinuses. Second, we compared the response to mepolizumab on nasal symptoms and the findings of CT scan opacification of paranasal sinuses between patients with AERD and those without AERD. Next, we assessed the changes of $\mathrm{FEV}_{1}$ with mepolizumab treatment. Finally, we investigated whether mepolizumab possess oral corticosteroid-sparing effect in the patients.

\section{Patients and methods}

\section{Patients}

The study population comprised 11 subjects ( 6 males and 5 females), median age 55.0 years in the age range 29 - 69 years in males, and median age 50.4 years in the age range $44-56$ years in females, respectively. The diagnosis of bronchial asthma was confirmed based on the Global Initiative for Asthma (GINA) guidelines (18). Enrolled patients in this study were required to have received a clinical diagnosis of bronchial asthma by experienced pulmonologists. All patients showed that $\mathrm{FEV}_{1}$ measured with a spirometer was less than $80 \%$ of the predicted value for age, sex, and height, with documented short-acting $\beta_{2}$ agonist reversibility of more than $12 \%$ after administration of $180 \mu \mathrm{g}$ of salbutamol. Six patients ( 3 each in males and females) were AERD, who were diagnosed as reported (19). Four patients ( 3 males and 1 female, and 1 AERD male) had been receiving maintenance treatment with oral corticosteroids ( 5 to $10 \mathrm{mg}$ per day of prednisone or its equivalent) for at least 6 months before entering the study.

The diagnostic guidelines established by the American Academy of Otorhinolaryngology - Head and Neck Surgery were met in each patient for the diagnosis of CRS (20). All patients had been diagnosed with the presence of nasal polyps using a nasal endoscope by experienced otolaryngologists at other hospitals before the treatment. Then, they had undergone a pretreatment CT scan of paranasal sinuses, and diagnostic evidence of CRS was defined by experienced radiologist of our hospital using the Lund-Mackay (LM) scores (21). The Japanese Epidemiological Survey of Refractory Eosinophilic Chronic Rhinosinusitis (JESREC), which was a retrospective study conducted by 15 institutions in Japan, has subdivided CRS into non-eosinophilic and eosinophilic CRS using the JESREC score criteria (22), and the score was assessed from each patient in this study. Clinical characteristics of the study patients are shown in table $\mathbf{I}$.

All patients had to have experienced at least 2 asthma exacerbations in the previous year that were treated with systemic corticosteroids administered intravenously or orally for more than 3 days, or that required a visit to the emergency department and/or hospitalization. They were receiving treatment with an inhaled corticosteroid at high-dosage of more than $500 \mu \mathrm{g}$ fluticasone dry powder or equivalent daily dosage / long-acting $\beta_{2}$ agonists inhalers with an additional controller, for 12 months before enrollment. In addition, all patients had to have an eosinophil count at least $150 \mathrm{cells} / \mu \mathrm{l}$ in blood at screening or at least $300 \mathrm{cells} / \mu \mathrm{l}$ at some time during the previous year. Patients were allowed to continue their current therapy throughout the study. The exclusion criteria included present smoking, a past history of smoking greater than 10 pack-years, parasitic infection in the 6 months before study entry, substantial uncontrolled co-morbidity, possibility of pregnancy, and history of poor treatment adherence.

Mepolizumab $100 \mathrm{mg}$ was administered subcutaneously at baseline (visit 1; week 0 ), and then every 4 weeks for a total 48 weeks as an add-on to appropriate standard care that could be adjusted at the physician's discretion. Thirteen visits were completed to 48 weeks. Patients were asked about exacerbations at every 4-week clinic visit from baseline to week 48 (exit visit). Safety was evaluated at each visit by assessment of adverse events, vital signs and electrocardiographic findings along with clinical labo-

Table I - Clinical characteristics of the study patients.

\begin{tabular}{lcc}
\hline gender & male & female \\
\hline number of patients & 6 & 5 \\
\hline age (years) (median) & 55.0 & 50.4 \\
\hline allergic & 2 & 0 \\
\hline non-allergic & 4 & 5 \\
\hline duration of asthma (years) (mean) & 16.7 & 18.6 \\
\hline aspirin hypersensitivity (AERD) & 3 & 3 \\
\hline oral corticosteroid-dependent & 3 & 1 \\
\hline
\end{tabular}

AERD, aspirin-exacerbated respiratory disease. 
ratory testing variables at baseline (week 0 ) and at weeks 24 and 48. Blood eosinophil count was assessed from baseline and every 4 weeks until week 48 . FEV 1 was measured at baseline and at weeks 24 and 48 (exit visit).

This study was performed in accordance with Good Clinical Practice guidelines, and the ethics principles outlined in the Declaration of Helsinki 2008, and approved by the Institutional Ethics Committee of Sutoh Hospital (IRB\#20160051). Written informed consent was obtained from each individual before the study commenced. This study was conducted between June 2016 and December 2018.

\section{Clinical measurements}

Eosinophils in peripheral blood were counted automatically using a counter (Beckman Coulter, Fullerton, CA, USA) and MAXM A/L system (Beckman Coulter). Serum levels of total immunoglobulin $\mathrm{E}$ (IgE) were measured using the CAP system (Phadia, Uppsala, Sweden). The percentages of predicted FEV were measured using a spirometer (FUKUDA-77, Fukuda Denshi, Tokyo, Japan), and the best of 3 expirations was recorded.

The Sino-Nasal Outcome Test (SNOT-22) questionnaire (23) is a modification of a pre-existing instrument, the SNOT-20 (24) with 2 additional questions about anosmia and nasal congestion. The SNOT-22 is a validated questionnaire quantifying upper respiratory tract symptoms. Each subject completed the SNOT-22 by answering all questions based on a $0-5$ scale, where 0 defines no problems with the given symptom and 5 defines maximal problems. The scores range from 0 to 110 , with high scores indicating greater symptoms, and a change of 8.9 or more points represents a minimally important difference (23). In this study, each subject completed the SNOT-22 at baseline and at week 48 .

The findings of CT scan opacification of paranasal sinuses in each patient was blindly staged by the same radiologist using the LM score system at baseline and at week 48. In the scoring system, each paranasal sinus (anterior ethmoid, posterior ethmoid, maxillary, frontal, and sphenoid sinus on the right and left sides) was assigned a score ( 0 for no opacification, 1 for partial opacification, and 2 for total opacification), and the ostiomeatal complex on each side was also assigned a score ( 0 for patent, 1 for partially obstructed, and 2 for completely obstructed). So, the total score ranges from 0 to 24 . An LM score less than 4 was classified as no CT abnormality, and an LM score greater than or equal to 4 was classified as $\mathrm{CT}$ abnormality, suspecting the presence of CRS.

The JESREC scoring system (22) assessed either unilateral or bilateral, the presence of nasal polyps, number of peripheral blood eosinophils, and dominant shadow of ethmoid sinuses in CT scans of paranasal sinuses. A JESREC score higher than or equal to 11 was determined as eosinophilic CRS (22). We evaluated the JESREC score from each patient before starting mepolizumab treatment.

\section{Statistical analyses}

Data are presented as mean \pm SD or numbers of observations, unless stated otherwise. Difference in study variables over time was analyzed using the Dunnett multiple comparison test. The Wilcoxon signed-rank test was used to compare paired data. All statistical analyses were performed using Microsoft Excel for Mac 2011. A p value $<0.05$ was considered significant.

\section{Results}

No patients failed to continue mepolizumab treatment because of adverse events, such as local injection site reactions and anaphylactic reactions, and none of them complained of headache or signs of nasopharyngitis. No clinically relevant trends were observed in vital signs, electrocardiographic findings, or clinical laboratory testing data. All patients continued to receive mepolizumab throughout the trial period without exacerbations. The mean of the JESRES score before starting mepolizumab treatment was 12.6 in the score range 11-17.

Blood eosinophil counts at baseline were $409.8 \pm 259.1$ (mean $\pm \mathrm{SD}$ ), and it showed a rapid and sustained reduction with mepolizumab (at weeks 4, 24 and 48, the counts were $82.9 \pm 42.3$, $55.7 \pm 60.9$ and $49.5 \pm 35.5$, respectively; each $\mathrm{p}<0.01$ ).

The total SNOT-22 scores decreased by 18.0 points $(\mathrm{p}<0.01$; figure 1, A). Symptom scores of anosmia (SNOT-22 question 21 , figure 1, B) and nasal congestion (SNOT-22 question 22, figure $1, \mathbf{C})$ decreased by 2.5 points $(\mathrm{p}<0.05)$, and decreased by 1.9 points $(\mathrm{p}<0.01)$. The LM score decreased by 3.0 points $(\mathrm{p}<0.01$; figure 1, D). In aspirin-tolerant patients, the total SNOT-22 scores (figure 2, A), the SNOT-22 question 21 score (figure 2, B), the SNOT-22 question 22 score (figure 2, C), and the LM score (figure 2, D) decreased by 15.2 points, 3.8 points, 2.0 points, and 3.6 points, respectively (each $\mathrm{p}<$ 0.01 ). In AERD patients, the total SNOT-22 scores (figure 3, A) and the LM score (figure 3, D) decreased by 20.3 points and 2.5 points (both $\mathrm{p}<0.05$ ), but not the SNOT-22 question 21 score (figure 3, B) and the SNOT-22 question 22 score (figure 3, C). Some patients did not provide a change of SNOT-22 and/or LM score after the period of observation (table II). Namely, among 6 AERD patients 2 patients did not provide a change of SNOT-22 score, and 3 patients did not provide a change of LM score change. On the other hand, a patient with allergic, oral corticosteroid-dependent asthma did not show a change of LM score.

$\mathrm{FEV}_{1}$ at week 24 and at week 48 was $73.3 \pm 8.4 \%$ and $73.9 \pm$ $8.8 \%$ respectively, and increased compared with $69.0 \pm 10.5 \%$ at baseline (both $\mathrm{p}<0.05$ ) (figure 4). 
Figure 1 - Change in SNOT scores and the Lund-MacKay CT scorings before mepolizumab therapy ( 0 week) and 48 weeks after start of the therapy in 11 patients. A significant reduction of total SNOT-22 scores and the CT scorings was seen at week 48 after mepolizumab treatment. Also, SNOT-22 Question 21 and 22 were significantly reduced with the treatment, indicating an improvement in sinonasal symptoms of the patients with mepolizumab treatment. A, total SNOT-22 scores; $\mathbf{B}$, SNOT-22 question 21 (smell / taste); C, SNOT-22 question 22 (nasal congestion); D, Lund-MacKay CT scorings. Gray horizontal lines represent group means. mepo: mepolizumab.

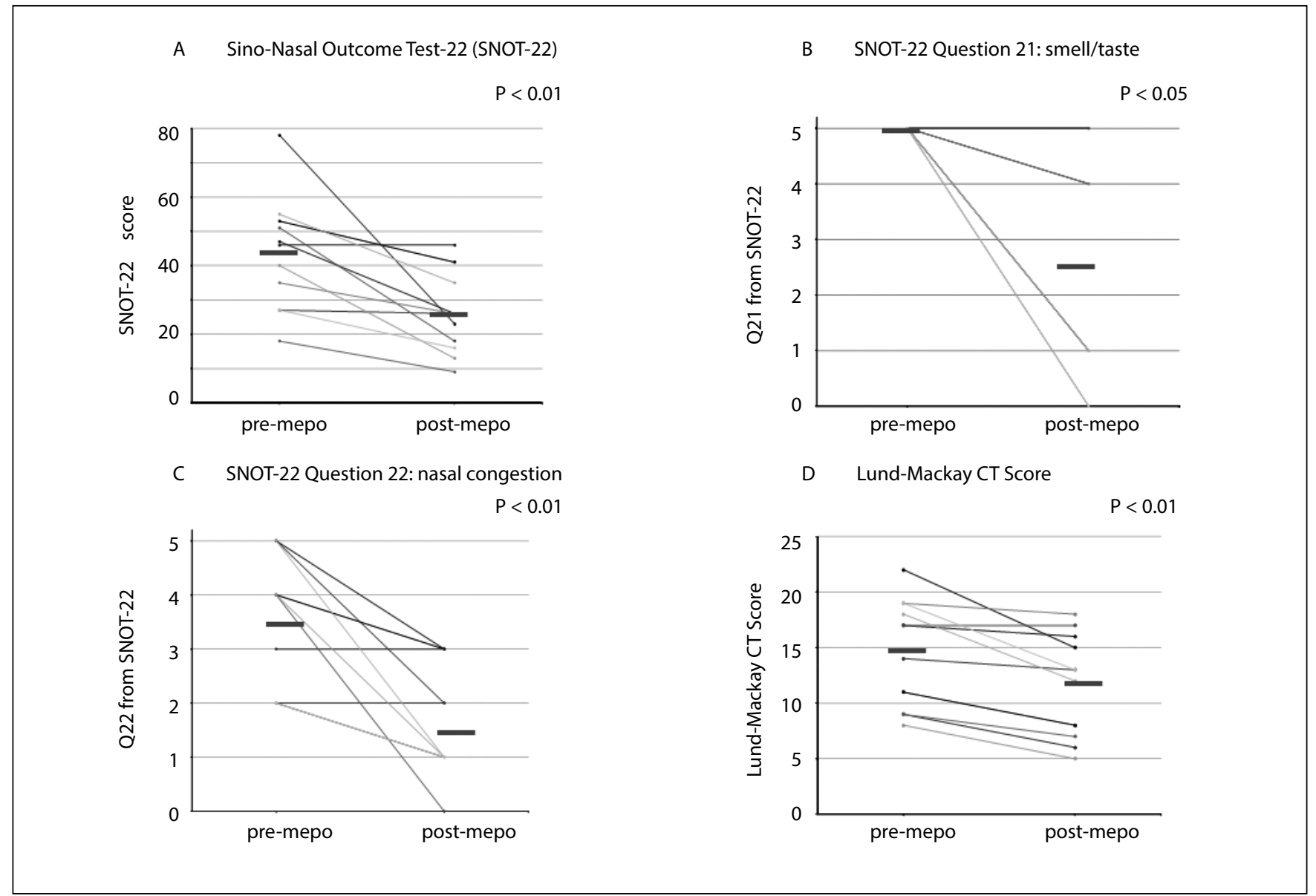

In the present study, 4 patients required daily oral corticosteroid therapy before starting the trial, and all of the patients successfully withdrew from daily use of oral corticosteroids without exacerbations and in parallel with sustained reduction in peripheral eosinophil count after initiation of the therapy (figure 5).

\section{Discussion}

This was the first open-label pilot study of subcutaneous administration of mepolizumab that showed safely improvements on nasal symptoms, the CT findings of the paranasal sinuses and lung function in Japanese patients of severe eosinophilic asthma with CRS. None of the patients experienced exacerbations during 48-week administration.
Before starting mepolizumab treatment, all patients had been diagnosed with the presence of nasal polyps using a nasal endoscope by experienced otolaryngologists at other hospitals. In addition, a JESREC score was assessed from each patient, and the mean JESREC score of the patients was 12.6 in the score range 11-17, supporting that all patients were eosinophilic CRS.

A recent review described blood eosinophil counts as a predictive biomarker for the efficacy of treatment with mepolizumab in patients with severe eosinophilic asthma (25). Following mepolizumab administration, a rapid and pronounced reduction in peripheral blood eosinophil levels was observed in this study, which was consistent with previous studies (25-27).

Unfortunately, after mepolizumab treatment less than half of the patients agreed to an endoscopic evaluation performed by expe- 
Figure 2 - Change in SNOT scores and the Lund-MacKay CT scorings before mepolizumab therapy ( 0 week) and 48 weeks after start of the therapy in 5 patients without aspirin hypersensitivity. A significant reduction of total SNOT-22 scores and the CT scorings was seen at week 48 after mepolizumab treatment. Also, SNOT-22 Question 21 and 22 was significantly reduced with the treatment. A, total SNOT22 scores; B, SNOT-22 question 21 (smell / taste); C, SNOT-22 question 22 (nasal congestion); D, Lund-MacKay CT scorings. Gray horizontal lines represent group means. mepo: mepolizumab.

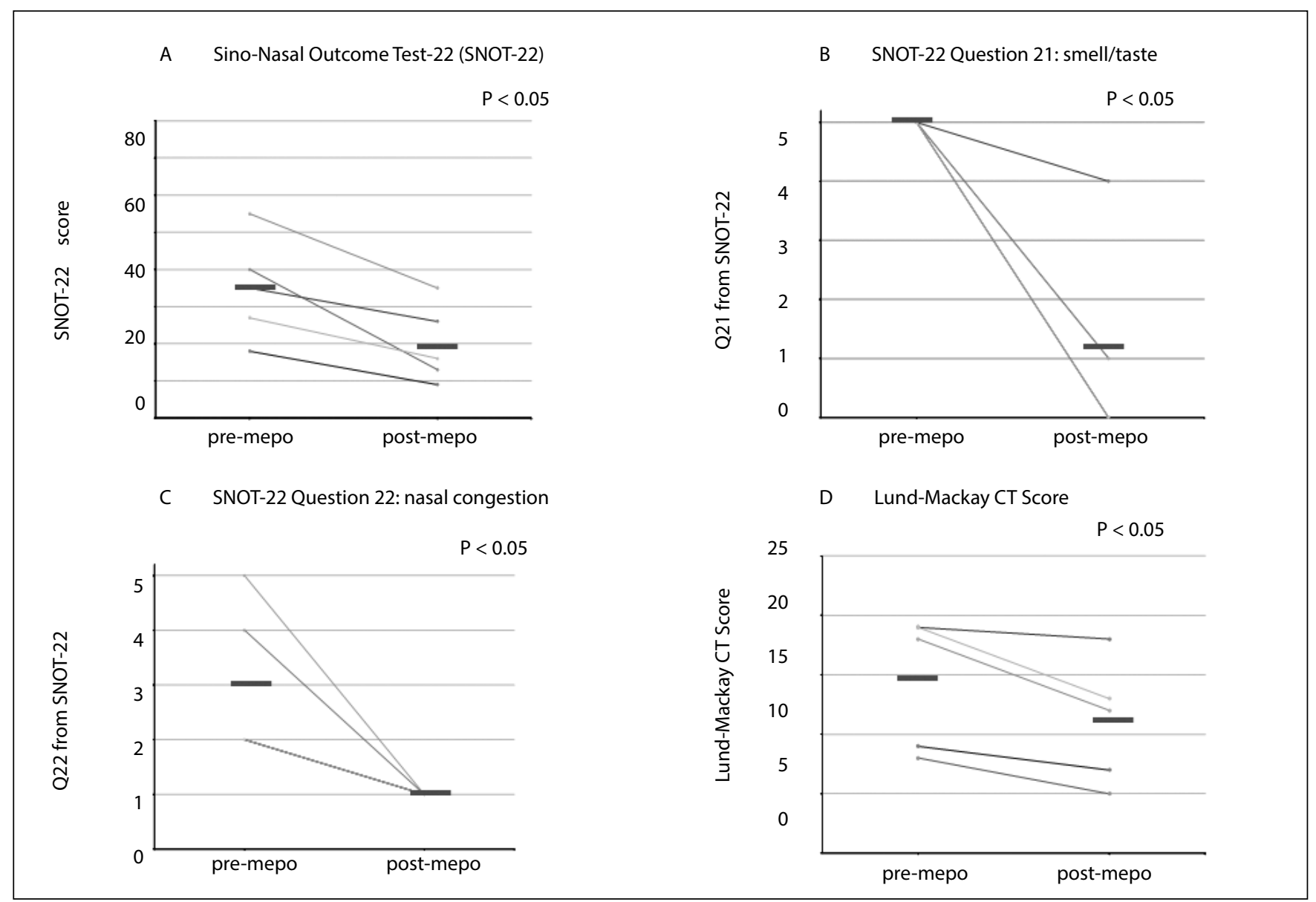

rienced otolaryngologists at other hospitals, and the rest of them refused to visit otolaryngologists. So, the primary objective of this study was to investigate whether mepolizumab may improve the symptoms of CRS and the findings of CT scan opacification of paranasal sinuses. Total SNOT-22 scores, symptom scores of anosmia (Question 21 from the SNOT-22) and nasal congestion (Question 22 from the SNOT-22) significantly decreased after the treatment. The next objective was the comparison of the response to mepolizumab on nasal symptoms and the CT findings between patients with AERD and aspirin-tolerant patients. In aspirin-tolerant patients, the total SNOT-22 scores, Question 21 score from the SNOT, Question 22 score from the SNOT-22, and the LM score significantly decreased with mepolizumab treatment. However, in AERD patients, the total SNOT-22 scores and the LM score significantly decreased, but not Question 21 and Question 22 scores from the SNOT-22. Some patients did not provide a change of SNOT-22 and/or LM score after the period of observation.

A recent investigation indicated that patients with AERD showed more olfactory loss, but no difference in the total SNOT-22, in comparison with patients without AERD (28). Peripheral blood eosinophil count has been shown to be higher in AERD patients than in aspirin-tolerant asthma $(19,29,30)$. On the other hand, it has been reported that while nasal congestion is a common symptom in patients with eosinophilic CRS, reduction in or loss of the sense of smell precedes nasal congestion (31), and characteristic CT images of the sinuses are opacification of posterior ethomoid sinus and the olfactory cleft $(22,32)$. Taking all into 
Figure 3 - Change in SNOT scores and the Lund-MacKay CT scorings before mepolizumab therapy ( 0 week) and at week 48 after start of the therapy in 6 patients with aspirin-exacerbated respiratory disease. A significant reduction of total SNOT-22 scores and the CT scorings was seen at week 48 after mepolizumab treatment. However, SNOT-22 Question 21 and 22 was not with the treatment. A, total SNOT22 scores; B, SNOT-22 question 21 (smell / taste); C, SNOT-22 question 22 (nasal congestion); D, Lund-MacKay CT scorings. Gray horizontal lines represent group means. mepo: mepolizumab, NS: not significant.

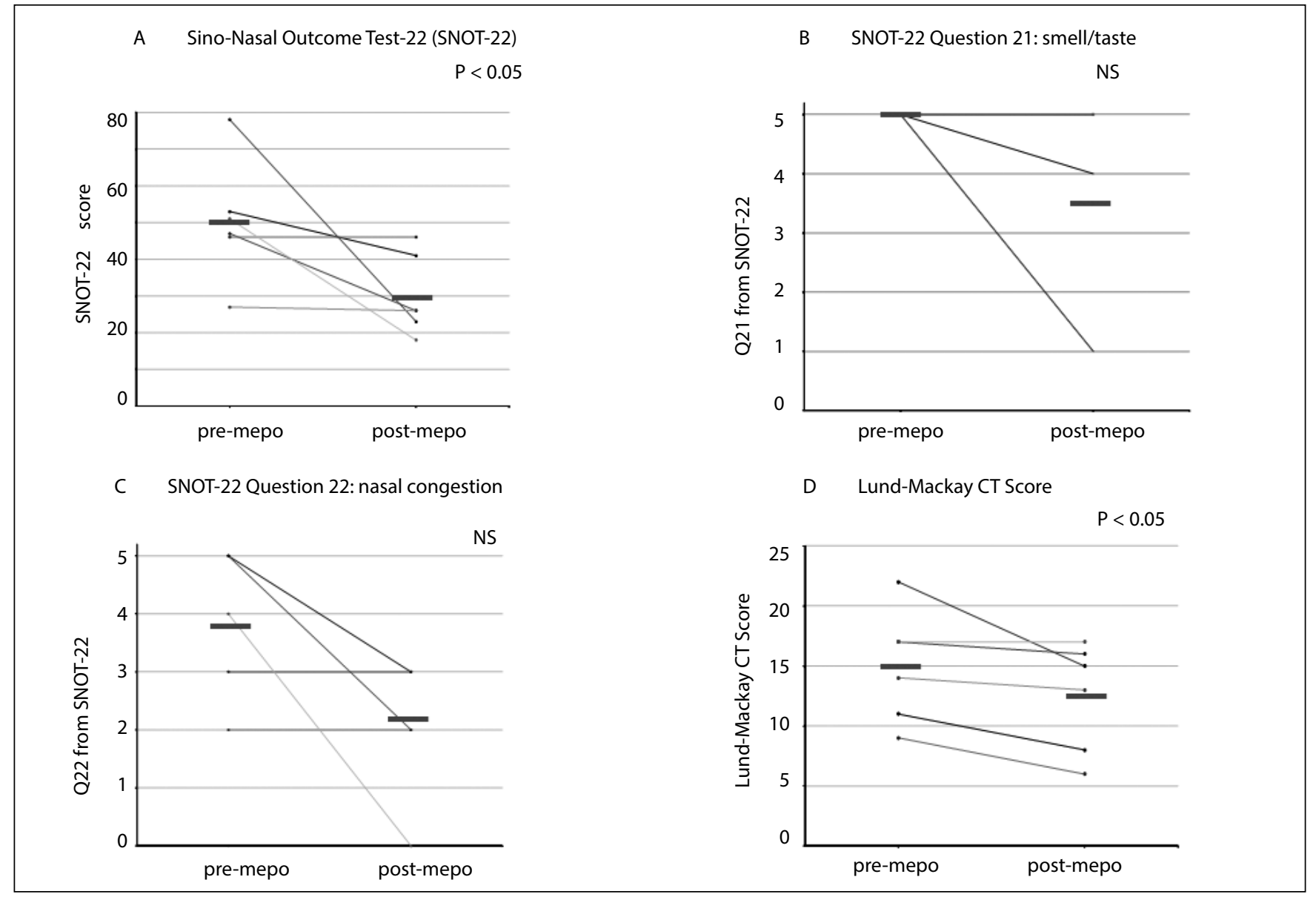

Table II - Patients and their clinical characteristics that did not provide changes of SNOT-22 score and/or Lund-Mackay CT score after mepolizumab treatment.

\begin{tabular}{lcccc}
\hline Patients (gender, age-years) & SNOT-22 score & \multicolumn{2}{c}{ Lund-Mackay CT score } \\
\hline & pre-mepo & post-mepo & pre-mepo & post-mepo \\
\hline patient 1 (female, 47, AERD) & 27 & 26 & 14 & 13 \\
\hline patient 2 (female, 44, AERD) & 46 & 46 & 9 & 17 \\
\hline patient 3 (female, 56, AERD) & 51 & 18 & 17 & 17 \\
\hline patient 4 (male, 46, AERD) & 47 & 26 & 19 & 18 (oral corticosteroid-dependent) \\
\hline patient 5 (male, 56, allergic) & 35 & 26 & & 16 \\
\hline
\end{tabular}

AERD, aspirin-exacerbated respiratory disease; mepo, mepolizumab. 
Figure 4 - Change in forced expiratory volume in one second $\left(F E V_{1}\right.$ ) before mepolizumab therapy ( 0 week), and 24 weeks, 48 weeks after start of the therapy. Significant improvements in FEV were seen at weeks 24 and at $48 .{ }^{*} p 0.05$.

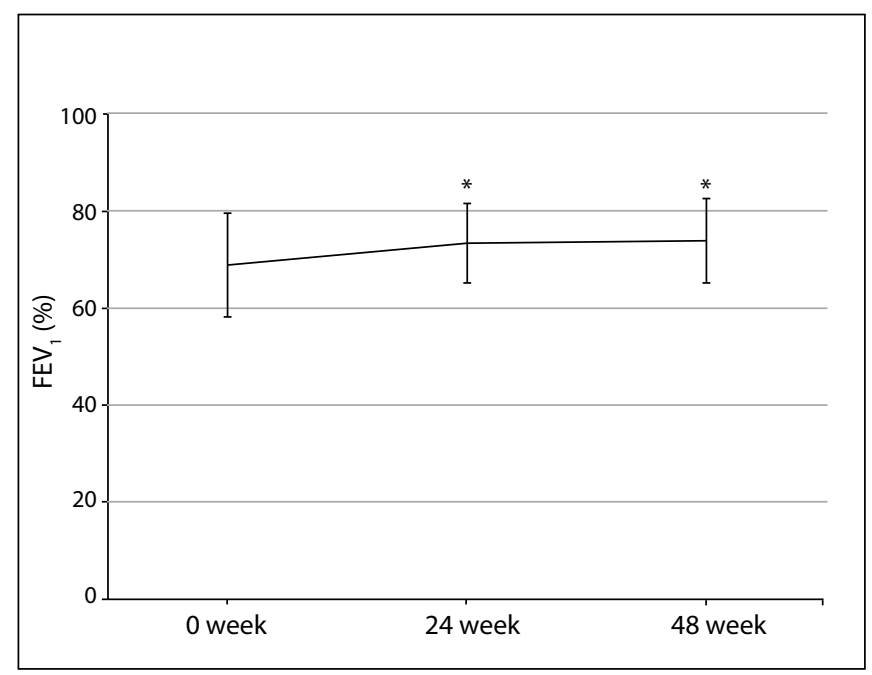

Figure 5 - Change in peripheral blood eosinophil count in oral corticosteroid-dependent asthma patients before mepolizumab treatment (week 0 ) and every 4 weeks thereafter. Solid lines show the eosinophil count under corticosteroid administration, and dotted lines show the eosinophil count without corticosteroids. All 4 corticosteroid-dependent asthma patients (3 aspirin-tolerant patients and 1 AERD patient) successfully withdrew from daily use of oral corticosteroids without exacerbations and in parallel with sustained reduction in peripheral blood eosinophil count after initiation of mepolizumab treatment. AERD: aspirin-exacerbated respiratory disease.

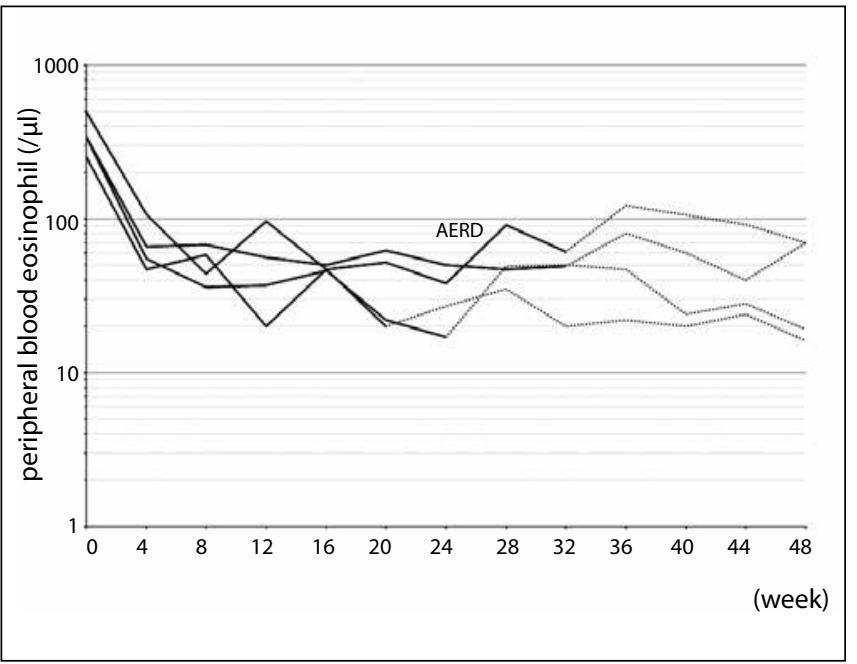

account, the findings in this study may suggest a possibility that local biologic activity of eosinophils, which induces symptoms of anosmia and nasal congestion, might be more severe in eosinophilic CRS with AERD, and longer duration of the treatment may be needed. Further studies are required.

In this study, some patients did not provide a change of SNOT-22 and/or LM score after the period of observation. Because this is a pilot study of 11 patients, we could not investigate their precise characteristic differences into responders and non-responders.

The Asthma Control Test (ACT) scores (33) is often used for assessment of asthma control. However, the ACT mainly depends on patient's reported outcome, and furthermore the presence of rhinitis has been shown to heavily affect the patient's perception of asthma control $(34,35)$. Some studies showed an evidence that rhinitis was associated with an incremental adverse impact on the disease-specific quality of life in asthmatic patients (34). Because the presence of rhinitis may affect the patient's perception of asthma, it was suggested that the accuracy of the ACT has not been systematically evaluated (36). Therefore, we assessed FEV in this study. The results showed $\mathrm{FEV}_{1}$ at week 24 and at week 48 were increased significantly compared with that at baseline. Finally, we evaluated the corticosteroid-sparing effect of meplozimab, because 4 patients required daily use of oral corticosteroids before initiating mepolizumab. All patients successfully withdrew from daily use of oral corticosteroids without exacerbation and in parallel with a sustained reduction in peripheral blood eosinophil count, which was consistent with the results of a previous report (37).

Needless to say, this pilot study has limitations. First, a modified LM CT system, which uses a 3-dimensional, computerized method to qualify the volume of mucosal inflammation in the sinuses, has been reported to better correlate with symptoms and disease-specific quality of life of the patients with CRS (38). However, the decrease of SNOT-22 scores was associated with that of LM scorings in the present study. Second, nasal IL-5 levels have been shown to determine the response to anti-IL-5 treatment in patients with nasal polyps (11). In this study, local biologic activity assessment and endoscopic evaluation could not be performed, because no experienced otolaryngologists work at our hospital. As concerns to nasal polyps, investigations about changes of polyp sizes before and after mepolizumab treatment will be required. In addition, number of subjects in the study was 11 , and this trial was not randomized, blinded, nor placebo-controlled. So, multicenter, double-blinded, controlled studies are necessary to confirm our data.

\section{Conclusions}

Our results showed a favorable long-term safety and clinical efficacy of mepolizumab on the upper airway symptoms and lung function in severe eosinophilic asthma patients with CRS. 


\section{Acknowledgements}

The authors would like to thank Junya Maehata, BSc for his assistance in assembling figures, and Kenji Satoh, MPharm for his assistance in statistical analysis.

\section{Conflict of interest}

The authors declare that they have no conflicts of interest.

\section{Ethical disclosures}

Institutional ethics committee approved this study and written informed consent from each individual was obtained before the study.

\section{References}

1. Braunstahl GJ, Overbeek SE, Kleinjan A, Prins JB, Hoogsteden HC, Fokkens WJ. Nasal allergen provocation induces adhesion molecule expression and tissue eosinophilia in upper and lower airways. J Allergy Clin Immunol 2001; 107(3): 469-476.

2. Ponikau JU, Sherris DA, Kephart GM, Kern EB, Gaffey TA, Tarara JE, et al. Features of airway remodeling and eosinophilic inflammation in chronic rhinosinusitis: is the histopathology similar to asthma? J Allergy Clin Immunol 2003; 112(5):877882.

3. Leung DYM, Nelson HS, Szefler SJ, Busse WW. One airway disease-chronic rhinosinusitis and asthma. J Allergy Clin Immunol 2003; 112(5):818.

4. Bachert C, Vignola AM, Gevaert P, Leynaert B, Van Cauwenberge $\mathrm{P}$, Bousquet J. Allergic rhinitis, rhinosinusitis, and asthma: one airway disease. Immunol Allergy Clin North Am 2004; 24(1):19-43.

5. Hamilos DL. Chronic sinusitis. J Allergy Clin Immunol 2000; 106(2):213-227.

6. Fokkens WJ, Lund VJ, Mullol J, Bachert C, Alobid I, Baroody E, et al. EPOS 2012: European position paper on rhinosinusitis and nasal polyps 2012. A summary for otorhinolaryngologists. Rhinology 2012; 50(1):1-12.

7. Peters AT, Spector S, Hsu J, Hamilos DL, Baroody FM, Chandra RK, et al. Diagnosis and management of rhinosinusitis: a practice parameter update. Ann Allergy Asthma Immunol 2014; 113(4):347-385.

8. Hamilos DL, Leung DY, Wood R, Cunningham L, Bean DK, Yasruel $\mathrm{Z}$, et al. Evidence for distinct cytokine expression in allergic versus nonallergic chronic sinusitis. J Allergy Clin Immunol 1995; 96(4):537-544.

9. Suzuki M, Watanabe T, Suko T, Mogi G. Comparison of sinusitis with and without allergic sinusitis: characteristics of paranasal sinus effusion and mucosa. Am J Otolaryngol 1999; 20(3):143-150.

10. Sejima T, Holtappels G, Kikuchi H, Imayoshi S, Ichimura K, Bachert C. Cytokine profiles in Japanese patients with chronic rhinosinusitis. Allergol Int 2012; 61(1):115-122.

11. Baba S, Kagoya R, Kondo K, Suzukawa M, Ohta K, Yamasoba T. T-cell phenotypes in chronic rhinosinusitis with nasal polyps in Japanese patients. Allergy Asthma Clin Immunol 2015; 11:33. doi: 10.1186/s13223-015-0100-2.
12. Sanderson CJ. Interleukin-5, eosinophils, and disease. Blood 1992; 79(12):3101-3109.

13. Gevaert P, Lang-Loidolt D, Lackner A, Stammberger H, Staudinger H, Van Zele T, et al. Nasal IL-5 levels determine the response to anti-IL-5 treatment in patients with nasal polyps. J Allergy Clin Immunol 2006; 118(5):1133-1141.

14. Gevaert P, Van Bruaene N, Cattaert T, Van Steen K, Van Zele T, Acke F, et al. Mepolizumab, a humanized anti-IL-5 mAb, as a treatment option for severe nasal polyposis. J Allergy Clin Immunol 2011; 128(5):989-995.

15. Bachert C, Sousa AR, Lund VJ, Scadding GK, Gevaert P, Nasser $S$, et al. Reduced need for surgery in severe nasal polyposis with mepolizumab: randomized trial. J Allergy Clin Immunol 2017; 140(4):1024-1031.

16. Kennedy JL, Stoner AN, Borish L. Aspirin-exacerbated respiratory disease: prevalence, diagnosis, treatment, and considerations for the future. Am J Rninol Allergy 2016; 30(6):407-413.

17. Tuttle KL, Buchheit KM, Laidlaw TM, Cahill KN. A retrospective analysis of mepolizumab in subjects with aspirin-exacerbated respiratory disease. J Allergy Clin Immunol Pract 2018; 6(3):1045-1047.

18. Global Initiative for Asthma (GINA). Global strategy for asthma management and prevention. http://www.ginasthma.org/local/uploads/files/GINA_Report_2015. Accessed May 2015.

19. Kurosawa M, Yukawa T, Hozawa S, Mochizuki H. Recent advances in investigation of gene polymorphisms in Japanese patients with aspirin-exacerbated respiratory disease. Allergol Immunopathol (Madr) 2015; 43(1):92-100.

20. Lanza DC, Kennedy DW. Adult rhinosinusitis defined. Otolaryngol Head Neck Surg 1997; 117(3 Pt 2):S1-7.

21. Lund VJ, Mackay IS. Staging in rhinosinusitis. Rhinology 1993; 31(4):183-184.

22. Tokunaga T, Sakashita M, Haruna T, Asaka D, Takeno S, Ikeda H, et al. Novel scoring system and algorithm for classifying chronic rhinosinusitis: the JESREC Study. Allergy 2015; 70(8):995-1003.

23. Hopkins C, Gillett S, Slack, Lund VJ, Browne JP. Psychometric validity of the 22-item Sinonasal Outcome Test. Clin Otolaryngol 2009; 34(5):447-454.

24. Piccirillo JF, Meritt MG Jr, Richards ML. Psycometric and clinimetric validity of the 20-Item Sino-Nasal Outcome Test (SNOT20). Otolaryngol Head Neck Surg 2002; 126(1):41-47.

25. Yancey SW, Keene ON, Albers FC, Ortega H, Bates S, Bleecker ER, et al. Biomarkers for severe eosinophilic asthma. J Allergy Clin Immunol 2017; 140(6):1509-1518.

26. Leckie MJ, ten Brinke A, Khan J, Diamant Z, O'Connor BJ, Walls CM, et al. Effect of an interleukin-5 blocking monoclonal antibody on eosinophilic airway hyper-responsiveness and the late asthmatic response. Lancet 2000; 356(9248):2144-2148.

27. Pavord ID, Korn S, Howarth P, Bleecker ER, Buhl R, Keene ON, et al. Mepolizumab for severe eosinophilic asthma (DREAM): a muliticentre, double-blind, placebo-controlled trial. Lancet 2012; 380(9842):651-659.

28. Gudziol V, Michel M, Sonnefeld C, Koschel D, Hummel T. Olfaction and sinonasal symptoms in patients with CRSwNP and AERD and withour AERD: a cross-sectional and longitudinal study. Eur Arch Otorhinolaryngol 2017; 274(3):14871493.

29. Sampson AP, Cowburn AS, Sladeck K, Adamek L, Nizankowska E, Szczeklik A, et al. Profound overexpression of leukotriene C4 
synthase in bronchial biposies from aspirin-intolerant asthmatic patients. Int Arch Allergy Immunol 1997; 113(1-3):355-357.

30. Szczeklik A, Nizankowska E, Duplaga M. Natural history of aspirin-induced asthma. AIANE Investigators. European network on aspirin-induced asthma. Eur Respir J 2000; 16(3):432-436.

31. Ishitoya J, Sakuma Y, Tsukuda M. Eosinophilic chronic rhinosinusitis in Japan. Allergol Int 2010; 59(3):239-245.

32. Sakuma Y, Ishitoya J, Komatsu M, Shiono O, Hirama M, Yamashita $\mathrm{Y}$, et al. New clinical diagnostic criteria for eosinophilic chronic rhinosinusitis. Auris Nasus Larynx 2011; 38(5):583-588.

33. Jia CE, Zhang HP, Lv Y, Liang R, Jiang YQ, Powell H, et al. The Asthma Control Test and Asthma Control Questionnaire for assessing asthma control: systemic review and meta-analysis. J Allergy Clin Immunol 2013; 131(3):695-703.

34. Vandenplas O, Dramaix M, Joos G, Louis R, Michils A, Verleden $\mathrm{G}$, et al. The impact of concomitant rhinitis on asthma-related quality of life and asthma control. Allergy 2010; 65(10):12901297.

35. Bousquet J, Schunemann HJ, Samolinski B, Demoly P, Baena-Cagnani CE, Bachert $C$, et al. Allergic Rhinitis and its Impact on Asthma (ARIA): achievements in 10 years and future needs. J Allergy Clin Immunol 2012; 130(5):1049-1062.

36. Caminati M, Caimmi C, Dama A, Schiappoli M, Passalacqua G, Senna G. What lies beyond Asthma Control Test: Suggestions for clinical practice. J Asthma 2016; 53(6):559-562.

37. Bel EH, Wenzel SE, Thompson PJ, Prazma CM, Keene ON, Yancey $\mathrm{SW}$, et al. Oral glucocorticosteroid-sparing effect of mepolizumab in eosinophilic asthma. N Engl J Med 2014; 371(13):1189-1197.

38. Garneau J, Ramirez M, Armato SG III, Sensakovic WF, Ford MK, Poon CS, et al. Computer-assisted staging of chronic rhinosinusitis correlates with symptoms. Int Forum Allergy Rhinol 2015; 5(7):637-642. 